\title{
Investigação das correlações existentes entre o ciclo vigília- sono e a dor num modelo experimental de fibromialgia
}

\author{
Edison Sanfelice André * \\ Camila Naumann Pereira \\ Beatriz Ferreira Nunes \\ Universidade Regional de Blumenau, Departamento de Fisioterapia \\ Laboratório de Fisioterapia Neurológica Experimental \\ Rua São Paulo, 2171, 3ํAndar, A506-B, CEP 89.030-000, Blumenau - SC, Brasil \\ * Autor para correspondência \\ sanfelice@furb.br
}

Submetido em 02/06/2020

Aceito para publicação em 17/09/2020

\section{Resumo}

Os distúrbios do sono são altamente prevalentes em pacientes portadores de fibromialgia. Muitos dos sintomas diurnos, como sonolência excessiva diurna e fadiga, podem estar relacionados a padrões de sono não restaurador, que, associados aos quadros álgicos típicos da doença, se retroalimentam. Neste contexto, objetivamos estudar as possíveis correlações existentes entre o ciclo vigília-sono e os comportamentos álgicos em um modelo animal de fibromialgia induzido por injeções repetidas de reserpina. Foram utilizados ratos Wistar machos e fêmeas adultos que receberam implantes crônicos de eletrodos bipolares encefálicos e de EMG. Injeções de reserpina provocaram aumento na resposta à estimulação mecânica, detectada através do emprego do teste de von Frey. Nos ratos tratados com reserpina observou-se fragmentação do ciclo vigília-sono. Adicionalmente, observou-se a presença de comportamentos aberrantes acompanhados de modificações eletroencefalográficas notáveis quando comparados aos animais controle. Destaca-se, também, alterações em bandas de frequências específicas dos traçados de EEG correspondentes aos estados comportamentais de vigília atenta e de sono de ondas lentas. Por conta desses achados suspeita-se que o modelo de fibromialgia produzido por injeções de reserpina pode servir como modelo aplicável para esta doença em humanos.

Palavras-chave: EEG; Fibromialgia; Modelo animal; Reserpina; Sono

\section{Abstract}

Investigation of the correlations between the sleep-wake cycle and pain in an experimental model of fibromyalgia. Sleep disorders are highly prevalent in patients with fibromyalgia. Many of the daytime symptoms, such as excessive sleepiness and fatigue, can be related to non-restorative sleep patterns and associated with the typical pain conditions of the disease. In this context, we aim to study the possible correlations between the sleep-wake cycle and pain behavior in an animal model of fibromyalgia induced by repeated injections of reserpine. Male and female adult Wistar rats received chronic implants to record EEG and EMG activity. Injections of reserpine produced increases in the response to mechanical stimulation detected using the von Frey test. The reserpine treated rats showed increased incidence of the behavioral states of the sleep-wake cycle with concomitant reduction in the respective durations, showing fragmentation of the cycle. Additionally, the 
presence of aberrant behaviors was observed in these animals accompanied by notable electroencephalographic changes when compared to control animals. Also noteworthy are changes in specific frequency bands of the EEG corresponding to attentive wakefulness and slow-wave sleep states. Based on these findings, it is possible to infer that the reserpine model of fibromyalgia can be a useful model for this disease in humans.

Key words: Animal model; EEG; Fibromyalgia; Reserpine; Sleep

\section{Introdução}

A fibromialgia (FM) é um exemplo típico de síndrome dolorosa crônica cuja etiologia é pouco compreendida. Sua prevalência global é de aproximadamente $2,7 \%$, sendo mais frequente em mulheres e em indivíduos com níveis educacionais e socioeconômicos baixos (QUEIRÓZ, 2013). É caracterizada por dores musculoesqueléticas, distúrbios do sono, fadiga, depressão, ansiedade, comportamentos mal adaptados que ocorrem sem nenhuma causa aparente (DADABHOY; CLAUW, 2006; FITZCHARLES et al., 2013; KNIGHT et al., 2013). Apesar de a dor representar a sintomatologia primordial, os demais sintomas são igualmente importantes devido ao impacto provocado na qualidade de vida dos pacientes e nas repercussões socioeconômicas (CONSOLI et al., 2012).

A fisiopatologia da fibromialgia tem sido explorada em níveis clínicos e pré-clínicos, mas o mecanismo detalhado ainda permanece desconhecido (BUSKILA; SARZI-PUTTINI, 2006; DADABHOY et al., 2008). No entanto, baseado em exames de imagem, testes genéticos e inovadores testes experimentais de dor (CLAUW, 2009), sugere-se o estudo da etiologia da doença através de mecanismos periféricos (BENGTSSON; HENRIKSSON, 1989; GUPTA et al., 2007; KATZ et al., 2007) e centrais (SIMMS, 1996; LEGOFF, 2006). A ausência de evidências definitivas de uma patologia muscular periférica que justifique a síndrome e os demais sintomas (NIELSEN; HENRIKSSON, 2007; BRADLEY, 2009; CLAUW, 2009; MCCARBERG, 2012), principalmente após os estudos realizados por Moldofsky et al. (1975), indica que a fibromialgia pode se manifestar a partir de mecanismos fisiopatológicos hospedados em locais específicos do sistema nervoso central (MOLDOFSKY, 2009; SPAETH; RIZZI, 2011).

O estresse possui papel importante no desencadeamento dos sintomas álgicos e demais associados à fibromialgia, pois afeta o eixo hipotalâmico-pituitárioadrenal (HPA) e o sistema autônomo, que altera os níveis de cortisol, promovendo disfunção de hormônio do crescimento além de alterações no ciclo vigíliasono (DADABHOY et al., 2008; BRADLEY, 2009; CLAUW, 2009). A alteração experimental do sono de ondas lentas em pacientes saudáveis pode induzir dor musculoesquelética e sintomas de fadiga através da modulação do eixo HPA (MOLDOFSKY et al., 1975; MOLDOFSKY, 2009). Há ainda uma série robusta de evidências demonstrando que os sistemas reguladores da sensibilidade e do ciclo vigília-sono compartilham os mesmos circuitos neurais (NIEUWENHUYS et al., 1998). Observa-se, deste modo, a manifestação de distúrbios do sono em portadores de fibromialgia.

É comum pacientes com fibromialgia reclamarem da dificuldade em adormecer à noite, além de fadiga, sonolência e de sensação de sono não restaurador pela manhã. Adicionalmente, essas queixas são acompanhadas pelo recrudescimento da sensação dolorosa, indicando haver forte correlação entre elas (BIGATTI et al., 2008; HAMILTON et al., 2008; CHOY, 2015).

Anormalidades eletrofisiológicas na FM foram relatadas pioneiramente em 1975, caracterizadas pela presença de padrões alfa-delta (intrusão alfa) em registros eletroencefalográficos (EEG) durante o sono de pacientes (MOLDOFSKY, 1981). Essas frequências, que geralmente caracterizam o estado de vigília, substituem ondas delta do sono não REM e podem ser uma explicação para a fadiga diurna relatada por pacientes portadores da síndrome (CHERVIN et al., 2009).

Sistemas neuronais descendentes do tronco encefálico, relacionados à modulação da sensibilidade somática, apresentam redução nos níveis de serotonina (5HT) e noradrenalina (NA) no líquido cefalorraquidiano em portadores de fibromialgia (RUSSELL et al., 1992). Esses mesmos sistemas desempenham papel funcional 
importante na regulação de diferentes estados do ciclo vigília-sono (AVERY; KRICHMAR, 2017).

Tais sistemas podem ser estudados através do emprego de drogas agonistas e/ou antagonistas que modificam as funções a eles relacionadas. Entre essas drogas, destaca-se a reserpina. A reserpina causa depleção de aminas biogênicas (noradrenalina, dopamina e serotonina) no SNC por se ligar irreversivelmente às moléculas transportadoras de monoaminas (SCHULDINER et al., 1995).

No sistema nervoso central, a noradrenalina é um neurotransmissor produzido por neurônios cujos corpos celulares estão localizados no locus coeruleus, região do tronco encefálico que desempenha papel importante no desencadeamento de respostas ao estresse e possui outras funções modulatórias importantes sobre o telencéfalo (AVERY; KRICHMAR, 2017). A literatura pertinente revela que a noradrenalina é um importante neurotransmissor para a promoção de estados de vigília atenta e, dependendo da atividade do núcleo locus coeruleus, também atua na geração do sono paradoxal ou REM (GOTTESMANN, 2008). O bloqueio completo da atividade noradrenérgica no SNC produz intensa sedação (HUNSLEY; PALMITER, 2003; BERRIDGE et al., 2012).

A dopamina é um neurotransmissor sintetizado a partir da tirosina. Existem quatro principais vias dopaminérgicas, a saber: a nigroestriatal, importante para o controle do movimento; a mesolímbica e a mesocortical, que são importantes para o afeto, a emoção e a motivação; e a que se origina no núcleo arqueado do hipotálamo e projeta-se para a glândula hipófise, onde regula a secreção de hormônios hipofisários (AVERY; KRICHMAR, 2017). Além dessas funções, a dopamina interfere no ciclo vigília-sono, pois injeções de doses baixas de agonistas de receptores dopaminérgicos reduzem a vigília e aumentam o sono de ondas lentas e o sono paradoxal, enquanto doses maiores produzem efeito oposto (MONTI; MONTI, 2007; NAKAZAWA et al., 2015).

A serotonina (5-hidroxitriptamina ou 5-HT) é um neurotransmissor sintetizado a partir do aminoácido essencial triptofano. Os corpos celulares dos neurônios serotonérgicos são encontrados nos núcleos da rafe na região medial do tronco encefálico e ao redor deles, e estão envolvidos na regulação da atenção e em outras funções cognitivas complexas. Redução nos níveis de serotonina no sistema nervoso central está diretamente implicada na gênese da depressão (NAUTIYAL, HEN 2017), o principal distúrbio do humor encontrado na fibromialgia (BELLATO et al., 2012). O papel da 5-HT no ciclo vigília-sono é conhecido há tempos, sendo o mais evidente o desencadeamento da vigília e a inibição do sono paradoxal. Por outro lado, injeções de antagonistas de receptores serotonérgicos produz aumento significante de sono de ondas lentas (PORTAS et al., 2000; MONTI, 2011).

O desenvolvimento de modelos animais para a fibromialgia é altamente complexo por exigir que o modelo mimetize grande parte dos sintomas apresentados pelos pacientes (FRANGE et al., 2014). O uso destes é substancialmente importante para a melhor compreensão dos mecanismos de desenvolvimento e manutenção da patologia, bem como para a introdução de possíveis tratamentos (MOCHIZUCHI, 2004; JONES et al., 2006; DAMASCENO et al., 2009; ZEILHOFER et al., 2009; DESANTANA et al., 2013). Estes estudos animais apontam certa relação entre alteração do sono e dor (MACHADO et al., 2004; ANDERSEN et al., 2016), sendo que evidências apontam que a dor persistente pode influenciar diretamente nas fases do sono (CARLI et al., 1987), como por exemplo na amplitude das ondas delta e na duração do sono de ondas lentas (LEYS et al., 2013).

Tomando como base as considerações acima, resolvemos investigar, no modelo animal de fibromialgia sugerido por Nagakura et al. $(2009 ; 2012)$ - no qual ocorre redução drástica nas concentrações de serotonina, noradrenalina e dopamina no sistema nervoso central, resultando em alodinia tátil, redução do limiar nociceptivo e imobilidade no teste do nado forçado - as possíveis relações entre os comportamentos álgicos e a atividade eletroencefalográfica do ciclo vigília-sono de animais tratados com reserpina. 


\section{Material e Métodos}

\section{Sujeitos experimentais}

Foram utilizados 20 ratos adultos da linhagem Wistar (10 machos e 10 fêmeas), pesando entre de 300 e $500 \mathrm{~g}$, fornecidos pelo Biotério Central da Universidade Regional de Blumenau (FURB). Todos os procedimentos aqui descritos respeitaram os preceitos éticos de experimentação animal preconizados pelo Conselho Nacional de Controle de experimentação Animal (CONCEA) e aprovados pela Comissão de Ética no Uso de Animais da FURB (01/2016).

Os animais permaneceram durante sete dias em ambientação antes do início dos experimentos, sendo mantidos individualmente em gaiolas acondicionadas em uma estante isoladora ventilada para roedores (ALESCO $\left.{ }^{\circledR}\right)$, instalada em ambiente com temperatura constante $\left(22 \pm 2^{\circ} \mathrm{C}\right)$, com ciclo claro-escuro de 12/12 (07h00-19h00 claro sob luz artificial - duas lâmpadas fluorescentes com $36 \mathrm{~W}$ de potência cada), e tiveram acesso livre à ração balanceada e à água filtrada durante todo o processo.

Posteriormente, os animais foram aleatoriamente distribuídos em dois grupos contendo, cada um, cinco machos e cinco fêmeas. No grupo controle $(\mathrm{N}=10)$, os animais receberam injeções de solução salina num volume fixo de $0,3 \mathrm{~mL}$ durante três dias consecutivos no mesmo horário (12h00). No grupo experimental $(\mathrm{N}=10)$, os animais receberam injeções de reserpina (1 mg/kg, s.c., Sygma-Aldrich) durante três dias consecutivos, conforme protocolo descrito por Nagakura et al. (2009), sempre no mesmo horário (12h00). Ao final dos experimentos, os animais foram sacrificados por sobredosagem anestésica. As carcaças, então, foram congeladas e descartadas conforme procedimento padrão adotado pela FURB.

\section{Análise do comportamento álgico}

Os testes de von Frey (detecção de alodinia mecânica) foram realizados e, após 10 minutos, foram aplicadas injeções de reserpina ou salina, nos tempos correspondentes 30, 60, 120 e 240 minutos (para mais detalhes, vide FERREIRA et al., 1978), durante os três dias de injeções. A frequência de resposta de retirada da pata foi obtida através de 10 aplicações (duração de $1 \mathrm{~s}$ cada) do filamento de von Frey 0,6 g (VFH, Stoelting, Chicago, USA). O filamento $0,6 \mathrm{~g}$, por produzir em média $15 \%$ de frequência de retirada da pata, foi utilizado durante todo o trabalho por ser considerado um valor adequado para a avaliação da hiperalgesia mecânica (FERREIRA et al., 1978). As respostas comportamentais para os testes foram assim classificadas: 0 - sem resposta, o animal não realiza a retirada da pata após estimulação; 1 - resposta discreta, pouca descarga de peso sobre a pata; 2 - pata elevada, não há contato da pata com a superfície de apoio; 3 - pata elevada, animal lambe, morde ou chacoalha a pata estimulada.

\section{Análise eletrográfica e comportamental do ciclo vigília-sono}

Para a obtenção dos registros de eletroencefalograma (EEG), todos os animais receberam implante crônico de eletrodos bipolares 10 dias antes das injeções de reserpina ou salina. Esses eletrodos foram confeccionados com fio de liga de níquel-cromo, revestido com teflon, com diâmetro de $0,3 \mathrm{~mm}$.

Os animais foram anestesiados com uma associação de $0,1 \mathrm{~mL}(10 \mathrm{mg} / \mathrm{kg})$ de xilazina e $0,3 \mathrm{~mL} / 100 \mathrm{~g}$ $(75 \mathrm{mg} / \mathrm{kg}$ ) de peso corporal de cloridrato de cetamina por via intraperitoneal. Atingido o nível anestésico esperado (ausência de reflexo de retirada ao pinçamento da cauda), os animais foram fixados em um aparelho estereotáxico (Insight, mod. EFF 331). Ato contínuo, a superfície craniana foi tricotomizada seguida de uma incisão longitudinal de $1,5 \mathrm{~cm}$ da pele. $O$ crânio foi perfurado com fresa esférica de uso odontológico (0,5 mm de diâmetro), primeiramente em três pontos para fixação dos parafusos ancoradores (região posterior do frontal esquerdo, região anterior do parietal direito e região lateral direita do osso occipital), posteriormente sobre os pontos correspondentes à área neocortical A3 ( $\mathrm{AP}=-1,5 ; \mathrm{L}=3,0)$, e para introdução do eletrodo na área hipocampal CA1 $(\mathrm{AP}=-3,0 ; \mathrm{L}=1,5 ; \mathrm{H}=$ $-3,0)$ seguindo as coordenadas estereotáxicas descritas por Paxinos e Watson (1997). Finalmente, os animais receberam implante de um par de eletrodos na 
musculatura eretora do pescoço para aquisição da atividade eletromiográfica (EMG).

Concluída a fase de implantação, os eletrodos foram soldados a um conector que foi ancorado ao crânio com o auxílio de acrílico autopolimerizante de uso odontológico. Sete dias após a implantação dos eletrodos, os animais foram conectados a um sistema de aquisição e análise de sinais biológicos (BIOPAC, MP150-WSW) para a validação dos registros eletrográficos. Após a validação da preparação, foram feitos registros de 30 minutos, adquiridos sempre entre $11 \mathrm{~h} 00$ e $17 \mathrm{~h} 00$ diariamente.

As amostras de EEG e de EMG correspondentes aos diferentes estados do ciclo vigília-sono (vigília atenta, vigília relaxada, sono sincronizado fases I, II e III, e sono paradoxal; conforme TIMO-IARIA et al., 1970) referentes aos períodos pré e pós-injeção de salina/ reserpina foram submetidas à transformada rápida de Fourier (FFT) em janelas temporais de quatro segundos.

A análise comportamental foi realizada off-line, por inspeção visual dos traçados de EEG e das anotações realizadas durante os registros. Foram computadas a incidência e a duração de cada estado encontrado. Tais valores foram, então, inseridos em uma planilha eletrônica para análise estatística posterior.

\section{Análise estatística}

Foram realizados testes de distribuição normal dos dados (Shapiro-Wilk) para cada uma das planilhas, exceto a dos dados referentes à FFT, que por sua natureza não demanda outro tipo de análise que não a descritiva. Os resultados dos testes de von Frey e os valores referentes à duração e incidência dos diferentes estados comportamentais do ciclo vigília-sono foram submetidos, num primeiro momento, à estatística descritiva, seguida de comparação das médias (pré e pós-injeções de salina e de reserpina) através de comparações múltiplas não-paramétricas de KruskalWallis. O nível de significância estabelecido foi de $p<0,05$. Finalmente, os gráficos contendo os resultados da FFT foram analisados descritivamente, destacando a expressão das potências das diferentes frequências contidas em cada espectro.

\section{Resultados}

Dos 20 animais empregados neste estudo, 13 animais (seis do grupo controle e sete do grupo experimental) foram considerados aptos para as análises específicas de EEG. Os sete faltantes foram excluídos por conta de problemas na implantação dos eletrodos que impossibilitaram registros adequados. Nos considerados aptos, foram realizados 50 diferentes registros representando as situações (grupos) de pré- e pós-injeções de salina e de reserpina.

\section{Alodinia}

Ao ser aplicado o teste de von Frey durante os momentos pré-teste, i.e., durante a obtenção dos valores limiares basais de retirada da pata posterior direita, não foi possível observar nenhuma diferença estatística significante nas comparações realizadas entre os grupos. Esses resultados foram os esperados, uma vez que em nenhum dos grupos havia sido feita qualquer intervenção.

Os mesmos resultados se repetiram durante os testes realizados após a primeira injeção (de reserpina e/ou de salina). A incapacidade de se detectar diferenças significantes no primeiro dia de tratamento reforça a hipótese de que a reserpina não produz efeitos agudos, pelo menos no que se refere à alodinia.

$\mathrm{Na}$ sessão de testes realizada no segundo dia, correspondente às injeções de reserpina, foi possível observar aumentos na frequência da retirada da pata dos animais tratados com reserpina aos 120 minutos $([\mathrm{H}(3)=4,500000, \mathrm{p}=0,048038])$ e aos 240 minutos $([\mathrm{H}(3)=12,89809, \mathrm{p}=0,002087])$.

No terceiro e último dia das injeções de reserpina, foi possível observar aumentos significantes na frequência de retirada da pata. Observou-se diferenças significantes nos tempos de 30 minutos ([H (3) = $5,65116, \mathrm{p}=0,028738]), 60$ minutos $([\mathrm{H}(3)=7,62712$, $\mathrm{p}=0,012847]), 120$ minutos $([\mathrm{H}(3)=19,05882$, $\mathrm{p}=0,000373])$ e 240 minutos $([\mathrm{H}(3)=17,00000$, $\mathrm{p}=0,000639])$. Conjuntamente, esses resultados revelam que as injeções de reserpina produziram os efeitos esperados no que se refere à indução de respostas hiperálgicas. 


\section{Ciclo vigília-sono}

Durante análise dos períodos pré- e pós-injeção de salina e de reserpina, não se observou a expressão do sono paradoxal (Figura 1). Suspeita-se que o tempo de registro definido em $30 \mathrm{~min}$. cada um foi insuficiente para haver adaptação adequada dos animais às condições de registro. Apesar disso, as demais fases, tanto de vigília quanto de sono sincronizado, foram registradas. Destaca-se que não foi possível encontrar diferenças nas latências do primeiro episódio de sono sincronizado. $\mathrm{O}$ grupo pré-salina teve latência média de $606,4 \pm 300 \mathrm{~s}$, o grupo salina $613 \pm 355 \mathrm{~s}$, o grupo pré-reserpina $636,3 \pm 341 \mathrm{~s}$ e o grupo tratado com reserpina $1.003 \pm 664 \mathrm{~s}$. Apesar de não haver diferença estatística significante, é possível notar que o grupo tratado com reserpina apresentou tendência de aumento na latência em relação aos demais grupos.
Mediante comparações feitas entre os grupos, observaram-se reduções estatisticamente significantes: na duração da vigília relaxada nos grupos pós-salina e pós-reserpina em relação ao grupo pré-salina; na duração do sono sincronizado I do grupo pós-reserpina em relação aos grupos pré- e pós-salina; na duração do sono sincronizado II do grupo pós-reserpina em relação aos demais grupos; na duração do sono sincronizado III dos grupos pós-salina, pré- e pós-reserpina em relação ao grupo pré-salina bem como redução na duração da fase III do sono sincronizado do grupo pós-reserpina em relação aos grupos pré-salina e pré-reserpina. Não foram observadas diferenças na duração da vigília atenta (dados não apresentados).

Em quatro dos sete animais do grupo tratado com reserpina observou-se a presença de comportamentos que se denominou aberrantes, caracterizados por

FIGURA 1: Duração dos estados comportamentais. (*) Diferenças significantes em relação ao grupo pré-salina. (\#) Diferenças significantes em relação ao grupo pós-salina. (++) Diferenças significantes em relação ao grupo pré-reserpina. $\mathrm{p}<0,05$ (Kruskal-Wallis).

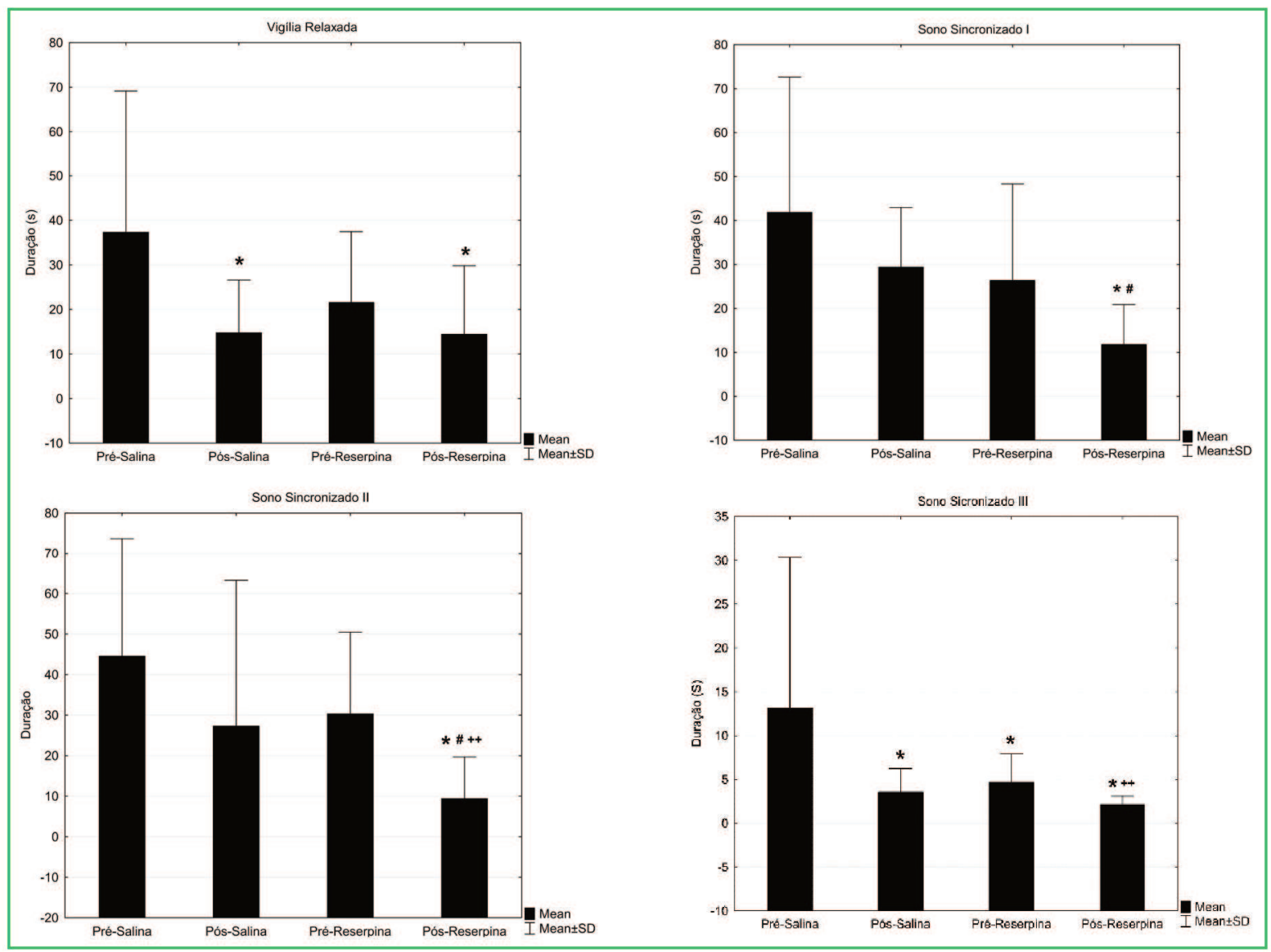


movimentos semelhantes a tremores e que são conhecidos como "wet dog shake" (parecidos com o chacoalhar que cães realizam para se secarem) e também por movimentos mastigatórios sem motivo aparente (vacuous chewing movements). A expressão desses comportamentos aconteceu com frequência aleatória a partir da terceira dose de reserpina e durou três dias, desaparecendo por completo após esse período. Esses comportamentos foram acompanhados de traçados eletrográficos peculiares que serão apresentados logo mais.

\section{Análise eletroencefalográfica}

Os traçados eletrográficos dos animais dos grupos pré-salina, pós-salina e pré-reserpina não demonstraram quaisquer alterações que mereçam destaque em nenhum dos estados comportamentais encontrados. O padrão do EEG da vigília atenta, da vigília relaxada, do sono sincronizado fases I, II e III preencheu os critérios de classificação descritos na literatura (VAN TWYVER, 1969; TIMO-IARIA et al., 1970).

No grupo tratado com reserpina, observou-se que, durante as fases I e II do sono sincronizado, ocorriam surtos de atividades rápidas na faixa de frequência acima de $10 \mathrm{~Hz}$ entremeando ondas lentas do tipo delta, o que poderia indicar a presença da alteração mais bem conhecida do EEG de portadores de fibromialgia que é a intrusão alfa (Figura 2).

Outro achado, destacado anteriormente, foram os comportamentos aberrantes que, além de se manifestarem de maneira específica e diferenciada, também revelaram um padrão de atividade eletrográfica igualmente diferenciado (Figura 3). Tais comportamentos geralmente eram precedidos de vigília atenta ou, hipoteticamente, distorcendo a vigília atenta. Por conta de terem ocorrido durante a vigília atenta, esperava-se encontrar atividade de EEG dessincronizada na área cortical A3 e presença de ritmo teta no hipocampo. Entretanto, o que se observou foram ondas lentas em grande amplitude em ambas as áreas, como é possível constatar nos histogramas dos espectros de frequência (Figura 3).
Interessantemente, esse tipo de atividade eletroencefalográfica da área cortical A3 não caracteriza nenhum estado comportamental do ciclo vigília-sono. Com o passar do tempo, por outro lado, essa atividade desapareceu e nenhum comportamento aberrante pôde ser encontrado.

\section{Discussão}

O presente estudo demonstrou que o modelo de fibromialgia sugerido por Nagakura et al. (2012) pode ser replicado, uma vez que foi possível observar aumentos na frequência de resposta em retirada da pata posterior durante os testes de von Frey. Foi possível demonstrar, também, e até onde foi possível avançar, achados inéditos na duração dos estados comportamentais de vigília relaxada, do sono sincronizado fases I, II e III e no registro eletroencefalográfico desses estados bem como o surgimento de comportamentos aberrantes decorrentes das injeções de reserpina.

Durante a fase referente à coleta de respostas em retirada da pata sem qualquer intervenção, não se observou diferença entre os diferentes grupos, resultado esperado para a situação em questão. No primeiro dia de injeção, também não foi possível detectar diferenças nas respostas de retirada da pata. A partir da segunda injeção, observaram-se diferenças significantes apontando que reduções mais intensas das aminas biogênicas iniciam após a segunda dose de reserpina, atingindo seu ápice após a terceira e última dose. Tomados em conjunto, esses resultados revelam que as injeções de reserpina produziram os efeitos esperados no que se refere à indução de respostas comportamentais hiperálgicas (alodinia) e estão em consonância com os dados da literatura pertinente (MOCHIZUCHI, 2004; NAGAKURA et al., 2009).

Apesar de não ter havido diferença estatística detectável, foi possível notar que o grupo tratado com reserpina apresentou tendência de aumento na latência do primeiro episódio de sono em relação aos demais grupos. Esse parece ser um indicativo de desorganização do ciclo vigília-sono encontrado nos casos de fibromialgia em humanos (e.g. HORNE; SHACKELL, 1991) e em modelos animais (DESANTANA et al., 2013; 
FIGURA 2: Trecho de $10 \mathrm{~s}$ referente ao sono sincronizado fase II de um animal tratado com reserpina. Em destaque nota-se alternância entre atividade rápida (acima de $10 \mathrm{~Hz}$ ) com atividade lenta (abaixo de $8 \mathrm{~Hz}$ ) na área cortical A3 conforme evidenciado no primeiro histograma do espectro de potência. O espectro de frequência referente à área hipocampal CA1 não revelou modificações. Na sequência do traçado nota-se uma aparente normalização rítmica característica desta fase do sono.

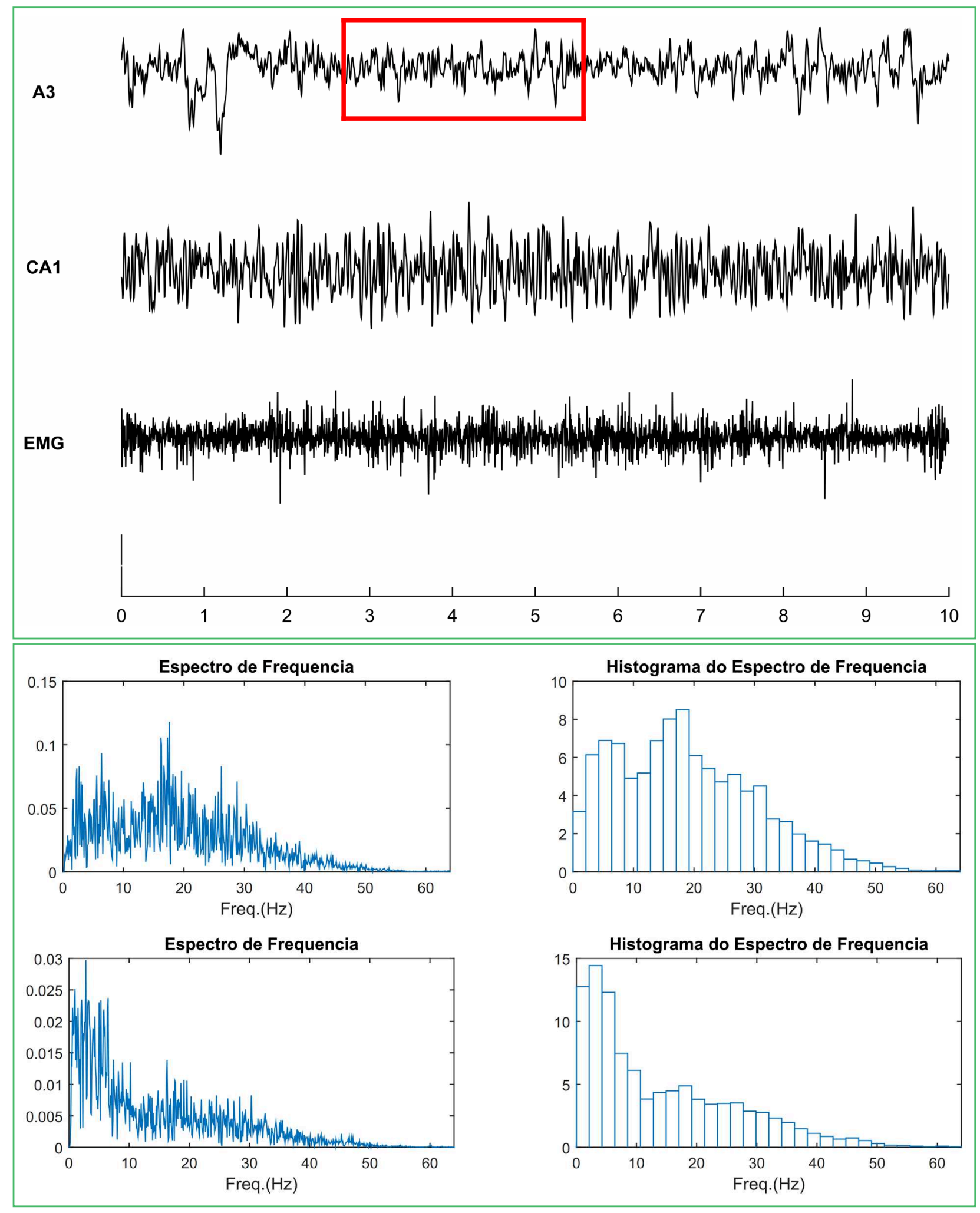


FIGURA 3: Imagem superior representando traçados eletrográficos de $20 \mathrm{~s}$ referentes ao denominado comportamento aberrante após o tratamento com reserpina. Logo abaixo, gráficos do espectro de frequência e o histograma das frequências dominantes da área cortical A3 e hipocampal CA1 respectivamente.

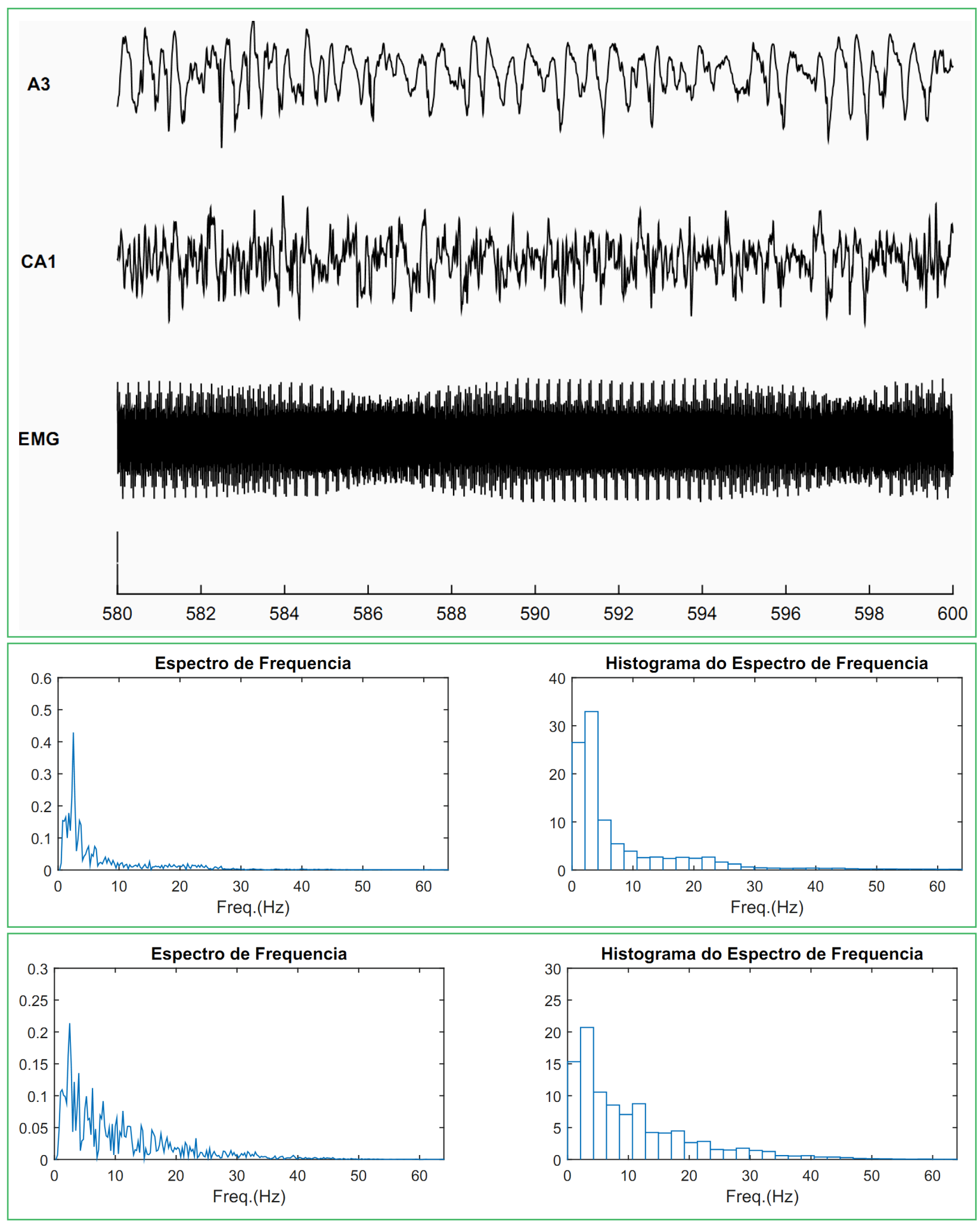


HERNANDEZ-LEON et al., 2019). Embora frágil, essa tendência sugere um efeito deletério das injeções de reserpina sobre o sono.

As observações comportamentais do ciclo vigília sono demonstraram que todos os estados comportamentais esperados estavam presentes exceto o sono paradoxal. Tal ausência pode ter ocorrido pela incapacidade de habituação dos animais aos procedimentos de registro dentro do tempo estipulado (30 minutos). Estudos pioneiros utilizando a reserpina para estudar o ciclo do sono em humanos demonstraram haver aumentos na duração do sono paradoxal com modificações discretas nas fases sincronizadas (HARTMAN, 1966; HOFFMAN; DOMINO, 1969) e também aumentos na incidência e duração do sono sincronizado fase I (e.g. JAIMCHARIYATAM et al., 2011). Entretanto, os critérios de alterações do sono em pacientes com fibromialgia, definidos pelo Colégio Americano de Reumatologia, incluem dificuldade em desencadear o sono, despertares constantes e dificuldade no despertar ao final do ciclo do sono (WOLFE et al., 2011). Ainda mais interessante é o fato de ratas ovariectomizadas e que receberam injeções de reserpina apresentaram abolição do sono paradoxal (HERNANDEZ-LEON et al., 2019). Essa evidência, apesar de ter sido encontrada em uma situação experimental diferente, pode justificar, especificamente, a ausência de sono paradoxal no grupo de animais tratados com reserpina. Assim, é prudente sugerir uma investigação mais profunda através da utilização de recursos que possam detalhar as fases do sono, como é o caso da polissonografia.

De maneira geral, reduções na duração de estados mais relaxados, como foi demonstrado neste trabalho, indicam níveis de estresse elevados, seja como resposta a distratores, seja por alerta constante. Uma possível justificativa para essas reduções poderia ser a manipulação dos animais que é exigida durante os diferentes procedimentos experimentais ou que precedia as sessões de registro (e.g., BALCOMBE et al., 2004). Entretanto, as reduções na duração das fases I e II do sono sincronizado e a aparente redução da fase III do sono sincronizado observadas no grupo pósreserpina quando comparado com o grupo pós-salina podem reforçar a ideia de a redução ter sido provocada diretamente pela droga com consequente desorganização do ciclo vigília-sono. Os animais tratados com reserpina, apesar de demonstrarem EEG compatível com vigília atenta, apresentaram redução de mobilidade na caixa de registro, permanecendo parados em um determinado local por longos períodos. Essa imobilidade reforça as evidências reveladas em outros estudos envolvendo a reserpina e seus efeitos sobre o comportamento geral de ratos (BASCO-SERRA et al., 2015; ZHEN-YONG et al., 2016).

Outro achado merecedor de destaque foi o surgimento de atividade eletrográfica enquadrada na faixa de frequências de $10 \mathrm{~Hz}$. Tal fenômeno, conhecido como intrusão alfa, aparece entremeando ritmos eletrográficos sincronizados (sono de ondas lentas) e apresenta correlação importante com os microdespertares noturnos (HORNE; SHACKELL, 1991) bem como com o sono de indivíduos portadores de transtornos de depressão, que também é um aspecto importante da síndrome da fibromialgia (JAIMCHARIYATAM et al., 2011). Acredita-se que essa atividade seja responsável pelas reduções na eficiência do sono (sono não restaurador), pela sonolência excessiva diurna e pelo recrudescimento da dor crônica na fibromialgia (HAMILTON et al., 2008; MOLDOFSKY, 2009).

Não menos importante foi o surgimento de comportamentos denominados aberrantes. O aparecimento do wet dog shake, num primeiro momento, causou espanto uma vez que tratamentos com reserpina costumam suprimir esse tipo de comportamento (COLASANTI et al., 1975; NABESHIMA et al., 1986). Por outro lado, esse comportamento em forma de tremor pode estar revelando uma complicação das injeções de reserpina que é a indução de parkinsonismo (DUTY; JENNER, 2011). Por sua vez, os vacuous chewing movements são característicos da síndrome denominada discinesia orofacial, e que também podem ser produzidos em ratos por injeções $(1 \mathrm{mg} / \mathrm{kg}) \mathrm{de}$ reserpina (PEREIRA et al., 2011). As doses empregadas no presente estudo foram as mesmas descritas na literatura, porém nem todos os animais apresentaram tais comportamentos aberrantes. Interessantemente, a atividade eletroencefalográfica observada na área cortical A3 e registrada durante os comportamentos aberrantes 
não é compatível com qualquer estado comportamental do ciclo vigília-sono. Com o passar do tempo, por outro lado, essa atividade desapareceu e nenhum comportamento aberrante pôde ser encontrado. Pensa-se que essa variante comportamental tenha ocorrido pelo efeito cumulativo das três doses de reserpina necessárias para produzir o modelo de fibromialgia anteriormente descrito. Entretanto, num único estudo encontrado durante as revisões da literatura, havia a descrição de atividade de EEG semelhante em ratos após injeção única de $0,5 \mathrm{mg} / \mathrm{kg}$ de reserpina em coelhos adultos (KUWANA, 1956).

Tomados em conjunto, esses achados, apesar de ainda preliminares, podem colaborar no entendimento dos mecanismos fisiopatológicos centrais da fibromialgia. Já está bem estabelecida a correlação entre fragmentação do sono e os quadros álgicos que se retroalimentam, porém, neste estudo, foram observadas não somente reduções na duração das fases do sono, mas também indícios de intrusão do ritmo alfa durante a fase II do sono sincronizado. Além disso, observou-se a expressão de comportamentos aberrantes, que também não foram descritos no estudo original que propôs o modelo de fibromialgia. Assim, sugere-se dar continuidade a esta linha de investigação utilizando ferramentas de análise de EEG que permitam processar registros mais longos e com maior riqueza de detalhes e o emprego de outras ferramentas que revelem uma possível interferência da reserpina na expressão de fatores neuroquímicos relacionados ao estresse, às condições de depressão/ ansiedade e à plasticidade neuronal no intuito de se desenvolver estratégias terapêuticas.

\section{Referências}

ANDERSEN, M. L.; NASCIMENTO, D. C.; MACHADO, R. B.; ROIZENBLATT, S.; MOLDOFSKY, H.; TUFIK, S. Sleep disturbance induced by substance $\mathrm{P}$ in mice. Behavioral Brain Research, Amsterdam, v. 167, n. 2, p. 212-218, 2016.

AVERY, M.C.; KRICHMAR, J.L. Neuromodulatory systems and their interactions: a review of models, theories and experiments. Frontiers in Neural Circuits, New York, v.11, n.118, p.1-18, 2017.

BALCOMBE, J. P.; BARNARD, N. D.; SANDUSKY, C. Laboratory routines cause animal stress. Contemporary Topics in Laboratory Animal Sciences, Memphis, v. 43, n. 6, p. 42-51, 2004.
BASCO-SERRA, A.; ESCRIHUELA-VIDAL, F.; GONZÁLEZSOLER, E. M.; MARTÍNEZ-EXPÓSITO, F.; BLASCO-AUSINA, M. C.; MARTÍNEZ-BELLVER, S.; CERVERA-FERRI, A.; TERUEL-MARTÍ, V.; VALVERDE-NAVARRO, A. A. Depressivelike symptoms in a reserpine-induced model of fibromyalgia in rats. Physiology \& Behavior, Oxford, v. 151. p. 456-462, 2015.

BELLATO, E.; MARINI, E.; CASTOLDI, F.; BARBASETTI, N.; MATTEI, L.; BONASIA, D. E.; BLONNA, D. Fibromyalgia Syndrome: etiology, pathogenesis, diagnosis, and treatment. Pain Research and Treatment, London, v. 4, n. 26, p. 1-17, 2012.

BENGTSSON, A.; HENRIKSSON, K. G. The muscle in fibromyalgia-a review of Swedish studies. The Journal of Rheumatology, Toronto, v. 16, n. 19, p. 144-149, 1989.

BERRIDGE, C. W.; SCHMEICHEL, B. E.; ESPAÑA, R. A. Noradrenergic modulation of wakefulness/arousal. Sleep Medicine Reviews, New York, v. 16, p. 187-197, 2012.

BIGATTI, S. M.; HERNANDEZ, A. M.; CRONAN, T. A.; RAND, K. L. Sleep disturbances in fibromyalgia syndrome: relationship to pain and depression. Arthritis \& Rheumatology, Hoboken, v. 59, p. 961-967, 2008.

BRADLEY, L. A. Pathophysiology of fibromyalgia. American Journal of Medicine, Tucson, v. 122, p. S22-30, 2009.

BUSKILA, D.; SARZI-PUTTINI, P. Genetic aspects of fibromyalgia syndrome. Arthritis Research \& Therapy, New York, v. 8, n. 5, p. 218-223, 2006.

CARLI, G.; MONTESANO, A.; RAPEZZI, S.; PALUFFI, G. Differential effects of persistent nociceptive stimulation on sleep stages. Behavioral Brain Research, Amsterdam, v.2 6, n. 2-3, p. 89-98, 1987.

CHERVIN, R. D.; TEODORESCU, M.; KUSHWAHA, R.; DELINE, A. M.; BRUCKSCH, C. B.; RIBBENS-GRIMM, C.; RUZICKA, D. L.; STEIN, P. K.; CLAUW, D. J.; CROFFORD, L. J. Objective measures of disordered sleep in fibromyalgia. The Journal of Reumathology, Toronto, v. 36, n. 9, p. 2009-2016, 2009.

CHOY, E. H. S. The role of sleep in pain and fibromyalgia. Nature Reviews in Rheumatology, Stuttgart, v. 11, p. 513-520, 2015.

CLAUW, D. J. Fibromyalgia: an overview. American Journal of Medicine, Tucson, v. 122, p. S3-S13, 2009.

COLASANTI, B. K.; KOSA, J. E.; CRAIG, C. R. Appearance of wet dog shake behavior during cobalt experimental epilepsy in rat and its suppression by reserpine. Psychopharmacologia, Heidelberg, v. 44, p. 33-36, 1975.

CONSOLI, G.; MARAZZITI, D.; CIAPPARELLI, A.; BAZZICHI, L.; MASSIMETTI, G.; GIACOMELLI, C.; ROSSI, A.; BOMBARDIERI, S.; DELL'OSSO, L. The impact of mood, anxiety, and sleep disorders on fibromyalgia. Comprehensive Psychiatry, Amsterdam, v. 1, n. 53, p. 962-967, 2012.

DADABHOY, D.; CLAUW, D. J. Therapy Insight: fibromyalgia - a different type of pain needing a different type of treatment. Nature, New York, v. 2, n. 7, p. 364-372, 2006.

DADABHOY, D.; CROFFORD, L. J.; SPAETH, M.; RUSSELL L. J.; CLAUW, D. J. Biology and therapy of fibromyalgia. Evidencebased biomarkers for fibromyalgia syndrome. Arthritis Research \& Therapy, New York, v. 8, n. 10, p. 211-228, 2008. 
DAMASCENO, F.; SKINNER, G. O.; GOMES, A.; ARAÚJO, P. C.; ALMEIDA, O. M. de Systemic amitriptyline administration does not prevent the increased thermal response induced by paradoxical sleep deprivation. Pharmacology Biochemistry and Behavior, Chilly-Mazarin, v. 9, n. 94-1, p. 51-55, 2009.

DESANTANA, J. M.; CRUZ, K. M. L.; SLUKA, K. A. Animal models of fibromyalgia. Arthritis Research \& Therapy, New York, v. 15, p. 222-235, 2013.

DUTY, S.; JENNER, P. Animal models of Parkinson's disease: a source of novel treatments and clues to the cause of the disease. British Journal of Pharmacology, London, v. 164, n. 4, p. 13571391, 2011.

FERREIRA, S. H.; ZANIN, T.; LORENZETTI, B. B.; SOUZA, M. Z. de; MEDEIROS, M. C.; LEME, J. G. Increased vascular permeability, oedema and hyperalgesia caused by carrageenin in the rat's paw proceedings. Agents Actions, Basel, v. 159, p. 81-92, 1978.

FITZCHARLES, M. A.; STE-MARIE, P. A.; PEREIRA, J. X. Fibromyalgia: evolving concepts over the past 2 decades. Canadian Medical Association Journal, Toronto, v. 185, n. 13, p. 645-651, 2013.

FRANGE, C.; HIROTSU, C.; HACHUL, H.; ARAUJO, P.; TUFIK, S.; ANDERSEN, M. L. Fibromyalgia and sleep in animal models: a current overview and future directions. Current Pain Headache Reports, Berlin, v. 18, n. 8, p. 434-444, 2014.

GOTTESMANN, C. Noradrenaline involvement in basic and higher integrated REM sleep processes. Progress in Neurobiology, Paris, v. 85, p. 237-272, 2008.

GUPTA, A.; MCBETH, J.; MACFARLANE, G. J.; MORRIS, R.; DICKENS, C.; RAY, D.; CHIU, Y. H.; SILMAN, A. J. Pressure pain thresholds and tender point counts as predictors of new chronic widespread pain in somatising subjects. Annals of the Rheumatic Diseases, London, v. 66, n. 4, p. 517-521, 2007.

HAMILTON, N. A.; AFFLECK, G.; TENNEN, H.; KARLSON, C.; LUXTON, D.; PREACHER, K. J.; TEMPLIN, J. L. Fibromyalgia: the role of sleep in affect and in negative event reactivity and recovery. Health Psychology, Washington, v. 27, n. 4, p. 490-497, 2008.

HARTMAN, E. Reserpine: its effect on the sleep-dream cycle in man. Psychopharmacologia, Heidelberg, v. 9, p. 242-246, 1966.

HERNANDEZ-LEON, A.; FERNANDEZ-GUÁSTI, A.; MARTÍNEZ, A.; PELLICER, F.; GONZÁLEZ-TRUJANO, M. E. Sleep architecture is altered in the reserpine-induced fibromyalgia model in ovariectomized rats. Behavioral Brain Research, Amsterdam, v. 364, p. 383-392, 2019.

HOFFMAN, J. S.; DOMINO, E. F. Comparative effects of reserpine on the sleep cycle of man and cat. Journal of Pharmacology and Experimental Therapeutics, Bethesda, v. 170, n. 2, p. 190-198, 1969.

HORNE, J. A.; SHACKELL, B. S. Alpha like-activity in non-REM sleep and the fibromyalgia (fibrositis) syndrome. EEG Clinical Neurophysiology, New York, v. 79, n. 4, p. 271-276, 1991.

HUNSLEY, M. S.; PALMITER, R. D. Norepinephrine-Deficient mice exhibit normal sleep-wake states but have shorter sleep latency after mild stress and low doses of amphetamine. Sleep, Oxford, v. 26, n. 5, p. 521-526, 2003.
JAIMCHARIYATAM, N.; RODRIGUEZ，C. L.; BUDUR, K. Prevalence and correlates of alpha-delta sleep in Major Depressive Disorders. Innovations in Clinical Neuroscience, West Chester, v. 8, n. 7, p. 35-49, 2011.

JONES, C. K.; EASTWOOD, B. J.; NEED, A. B.; SHANNON, H. E. Analgesic effects of serotoninergic, noradrenergic or dual reuptake inhibitors in the carrageenan test in rats: evidence for synergism between serotoninergic and noradrenergic reuptake inhibition. Neuropharmacology, Amsterdam, v. 51, v. 7-8, p. 1172-1180, 2006.

KATZ, D. L.; GREENE, L.; ALI, A.; FARIDI, Z. The pain of fibromyalgia is due to muscle hypoperfusion induced by regional vasomotor dysregulation. Medical Hypotheses, Amsterdam, v. 69, n. 3, p. 517-525, 2007.

KNIGHT, T.; SCHAEFER, C.; CHANDRAN, A.; ZLATEVA, G.; WINKELMANN, A.; PERROT, S. Health-resource use and costs associated with fibromyalgia in France, Germany, and the United States. Clinicoeconomics and Outcomes Research, Macclesfield, v. 5, p. 171-80, 2013.

KUWANA, S. The effects of reserpine and serpetin on the EEG in rabbits. Folia Pharmacologica Japonica, Tokyo, v. 52, n. 3, p. 317-321, 1956.

LEGOFF, P. Is Fibromyalgia a muscle disorder? Joint Bone Spine, Amsterdam, v. 73, n. 3, p. 239-242, 2006.

LEYS, L. J.; CHU, K. L.; XU, J.; PAI, M.; Yang, H. S.; ROBB, H. M.; JARVIS, M. F.; RADEK, R. J.; MCGARAUGHTY, $\mathrm{S}$. Disturbances in slow-wave sleep are induced by models of bilateral inflammation, neuropathic, and postoperative pain, but not osteoarthritic pain in rats. Pain, Washington, v. 154, n. 7, p. $1092-$ $1102,2013$.

MACHADO, R. B.; HIPÓLIDE, D. C.; BENEDITO-SILVA, A. A.; TUFIK, S. Sleep deprivation induced by modified multiple platform technique: quantification of sleep loss and recovery. Brain Research, Amsterdam, v. 1004, n. 1-2, p. 45-51, 2004.

MCCARBERG, B. H. Clinical overview of fibromyalgia. American Journal of Therapeutics, Alphen aan den Rijn, v. 19, n. 5, p. 357-368, 2012.

MOCHIZUCHI, D. Serotonin and noradrenaline reuptake inhibitors in animal models of pain. Human Psychopharmacology, Hoboken, v. 9, Suppl. 1, p. S15-19, 2004.

MOLDOFSKY, H. Rheumatic pain modulation syndrome: the interrelationships between sleep, central nervous system serotonin and pain. Advances in Neurology, Minneapolis, v. 33, p. 51-57, 1981.

MOLDOFSKY, $H$. The significance of the dysfunctions of the sleep-waking brain to the pathogenesis and treatment of fibromyalgia syndrome. Rheumatic Disease Clinics of North America, Amsterdam, v. 35, n. 2, p. 275-283, 2009.

MOLDOFSKY, H.; SCARISBRICK, P.; ENGLAND, R.; SMYTHE, H. Musculoskeletal symptoms and NonREM sleep disturbance in patients with 'fibrositis syndrom' and healthy subjects. Psychosomatic Medicine, Hoboken, v. 37, n. 4, p. 341351, 1975.

MONTI, J. M. Serotonin control of sleep-wake behavior. Sleep Medicine Reviews, New York, v. 15, p. 269-281, 2011.

MONTI, J. M.; MONTI, D. The involvement of dopamine in the 
modulation of sleep and waking. Sleep Medicine Reviews, New York, v. 11, p. 113-133, 2007.

NABESHIMA, T.; YAMAGUCHI, K.; YOSHIDA, S.; FURUKAWA, H.; KAMEYAMA, T. Phencyclidine-induced wet-dog shakes observed in rats after withdrawal from reserpine treatment. Pharmacology Biochemistry and Behavior, ChillyMazarin, v. 24, n. 5, p. 1275-1279, 1986.

NAGAKURA, Y.; OE, T.; AOKI, T.; MATSUOKA, N. Biogenic amine depletion causes chronic muscular pain and tactile allodynia accompanied by depression: a putative animal model of fibromyalgia. Pain, Washington, v. 146, n. 1-2, p. 26-33, 2009.

NAGAKURA, Y.; TAKAHASHI, M.; NOTO, T.; SEKIZAWA, T.; OE, T.; YOSHIMI, E.; TAMAKI, K.; SHIMIZU, Y. Different pathophysiology underlying animal models of fibromyalgia and neuropathic pain: comparison of reserpine-induced myalgia and chronic constriction injury in rats. Behavioral Brain Research, Amsterdam, v. 226, p. 242-249, 2012.

NAKAZAWA, S.; NAKAMICHI, K.; IMAI, H.; ICHIHARA, J. Effect of dopamine D4 receptor agonists on sleep architecture in rats. Progress in Neuro-Psychopharmacology \& Biological Psychiatry, Quebec, v. 63, n. 3, p. 6-13, 2015.

NAUTIYAL, K. M.; HEN, R. Serotonin receptors in depression: from A to B. F1000 Research, London, v. 6, n. 123, p. 1-12, 2017.

NIELSEN, L. A.; HENRIKSSON, K. G. Pathophysiologic mechanisms in chronic musculoskeletal pain (fibromyalgia): the role of central and peripheral sensitization and pain disinhibition. Best Practice \& Research: Clinical Rheumatology, Amsterdam, v. 21, n. 3, p. 465-480, 2007.

NIEUWENHUYS, R.; DONKELAAR, H. J. T.; NICHOLSON, C. The Central Nervous System of Vertebrates. Vol. 3. Berlin: Springer, $1998.960 \mathrm{p}$.

PAXINOS, G.; WATSON, C. The rat brain in stereotaxic coordinates. San Diego: Academic Press, 1997. 209 p.

PEREIRA, R. P.; FACHINETTO, R.; PRESTES, A. S.; WAGNER, C.; SUDATI, J. H.; BOLIGON, A. A.; ATHAYDE, M. L; MORSCH, V. M.; ROCHA, J. B. T. Valeriana officinalis ameliorates vacuous chewing movements induced by reserpine in rats. Journal of Neural Transmission, New York, v. 118, n. 11, p. 1547-1557, 2011.
PORTAS, C. M.; BJORVATN, B.; URSIN, R. Serotonin and the sleep/wake cycle: special emphasis on microdialysis studies. Progress in Neurobiology, Paris, v. 60, p. 13-35, 2000.

QUEIRÓZ, L. P. Worldwide epidemiology of fibromyalgia. Current Pain Headache Reports, New York, v. 17, n. 8, p. 356361, 2013.

RUSSELL, I. J.; VAEROY, H.; JAVORS, M.; NYBERG, F. Cerebrospinal fluid biogenic amine metabolites in fibromyalgia/ fibrositis syndrome and rheumatoid arthritis. Arthritis \& Rheumatology, London, v. 35, p. 550-556, 1992.

SCHULDINER, S.; SHIRVAN, A.; LINIAL, M. Vesicular neurotransmitter transporters: from bacteria to humans. Physiological Reviews, Washington, v. 75, n. 2, p. 369-392, 1995.

SIMMS, R. W. Is there muscle pathology in Fibromyalgia Syndrome? Rheumatic Disease Clinics of North America, Amsterdam, v. 22, n. 2, p. 245-266, 1996.

SPAETH, M.; RIZZI, M. Fibromyalgia and sleep. Best Practice \& Research Clinical Rheumatology, Amsterdam, v. 25, p. 227-239, 2011.

TIMO-IARIA, C.; NEGRÃO, N.; SCHMIDEK, W.; ROCHA, T. L.; HOSHINO, K. Phases and states of sleep in the rat. Physiology \& Behavior, Oxford, v. 5, p. 402-407, 1970.

VAN TWYVER, H. Sleep patterns of five rodent species. Physiology \& Behavior, Oxford, v. 4, p. 901-905, 1969.

WOLFE, F.; CLAUW, D. J.; FITZCHARLES, M. A.; GOLDENBERG, D. L.; HÄUSER, W.; KATZ, R. S.: MEASE, P.; RUSSELL, A. S.; RUSSELL, I. J.; WINFIELD, J. B. Fibromyalgia criteria and severity scales for clinical and epidemiological studies: a modification of the ACR preliminary diagnostic criteria for fibromyalgia. The Journal of Rheumatology, Toronto, v. 38, n. 6, p. 1113-1122, 2011.

ZEILHOFER, H. U.; MÖHLER, H.; DI LIO, A. GABAergic analgesia: new insights from mutant mice and subtype-selective agonists. Cell, London, v. 30, n. 8, p. 397-402, 2009.

ZHEN-YONG, G.; YANG, P.; QING-JUN, H.; HAI-YUN, X. The influence of dizocilpine on the reserpine-induced behavioral and neurobiological changes in rats. Neuroscience Letters, London, v. 614, p. 89-94, 2016. 